\title{
PARAMETER ESTIMATION BASED ON A SWITCHED OBSERVER FOR THE STUDY OF LEFT VENTRICLE CONTRACTILITY
}

\author{
Paolo Valigi \\ Department of Electronic and Information Engineering, \\ University of Perugia, \\ Via G. Duranti, 93 - Perugia, Italy \\ valigi@diei.unipg.it
}

\begin{abstract}
In this paper a method for estimating maximum ventricular elastance through a switched observer is proposed, based on measurement of ventricular volume (and possibly aortic pressure). The proposed switched observer, i.e, an observer for a switched system, is derived from an electrical-analog model of the left ventricle and systemic load. An observability study was a priori conducted on the model, restricted to the ejection phase, to validate the estimation procedure. The method has been evaluated with simulated data and produced good results. Copyright ${ }^{\complement} 2005$ IFAC.
\end{abstract}

Keywords: Parameter estimation, ventricle elastance, switched systems, observer.

\section{INTRODUCTION}

Ventricular elastance is recognized as a relevant index to measure ventricular contractility performance, mostly because of its independence on load conditions (Senzaki et al., 1996).

Estimation of cardiac functionality parameters has received large attention. In (Yoshizawa et al., 1998) a parameter optimization method is proposed, which regards the estimation process as an inverse problem, and is based on ventricular pressure measurement, together with either ventricular volume or ventricular outflow. This technique does not need any change in cardiac load, because the estimation is based on only information within one heart beat. The whole estimation process is based on two assumptions that the ventricular elastance is approximated with a linear function, and that at any time between the end-

\footnotetext{
1 This work was supported by MIUR under FIRB program, contract no. RBAU0123.
}

diastolic time and the beginning time of ejection, the ventricular volume can be represented as the difference between the end-diastolic volume and ejecting volume.

A different approach to maximum elastance estimation is proposed in (Yoshizawa et al., 1996), where a beat-by-beat estimation procedure is presented (Nitta and Yoshizawa, 1995), using measurements of aortic pressure and flow. The least square method is applied to an analog electrical circuit model of the systemic circulation, which holds under the assumptions that left ventricular elastance is approximated by a linear time function in the ejecting phase, and that the system dynamics from left ventricular pressure to aortic pressure are expressed by a series combination of a resistance and an inductance.

In (Guarini et al., 1998) a two-stages procedure for estimation of left ventricular parameters from the radial pressure waveform and cardiac output is presented. Aortic load is represented by a 
lumped-parameter transmission line model, whose parameters are sought in the first stage through an iterative process, based on sequential quadratic programming (SPQ) and the one-shot linear minimum squares (LMS). Using the radial pressure waveform, this procedure converges to a set of coefficients that are used later, together with cardiac output, to obtain the arterial load parameters. In the second stage these parameters are used to select the best fitting left-ventricular model out of four different possibilities, based on different analytical expressions for left-ventricular contractility, and to estimate ventricular parameter values.

A noninvasive method for on-line measurement of cardiac contractility is reported in (Ruggeri et al., 1997). The authors use a pressure-area diagram in place of the pressure- volume diagram to infer Elastance. The ventricle volume is derived from area measurements in a eco-cardiographic image, and intraventricular blood pressure is approximated with peripheral arterial pressure. A proper signal processing system is used in order to compensate the distortion and delay introduced in the pressure signal by its propagation from the ventricle to peripheral artery.

In (Kim et al., 1998) $E_{\max }$ estimation is based on ventricular volume and radial artery pressure measurements, and used to reconstruct a single pressure-volume loop, which is then used to estimate $E_{\max }$. Volume measurements are based on gated myocardial SPECT, whereas radial pressure is measured through an automated tonometer and then used to reconstruct control aortic pressure.

Estimation of parameters characterizing a ventricular mechanical performance, is considered also in (Karamanoglu and Feneley, 1996; Boom and Wijkstra, 1989; Kanai et al., 1999), where attention is given to parameters and/or performance indexes different from elastance and contractility.

Estimation of ventricular elastance is of major interest also in VAD application (Arabia et al., 2001) for destination therapy, where elastance is considered as a good candidate to manage weaning. Within this framework, the estimation of myocardium maximum elastance without direct measurement of the left ventricular volume is described in (Yoshizawa et al., 1994). The systemic circulation is represented by the three element Windkessel model and elastance time-dependence during the systolic phase is assumed sinusoidal. The key technique is to estimate the angular frequency identifying the value of the maximum elastance. Model parameters are determined via measurement of flow of the left ventricular assist device, and aortic pressure.

In (Nitta and Yoshizawa, 1995) the maximum elastance of the ventricle is approximated with the slope of the line by two end-systolic point on the left ventricular pressure-volume diagram and it is calculated from the difference of the left ventricular pressure -volume loop between two drive modes of Left Ventricular Assist Device (LVAD). Moreover the use of LVAD allows for further assumptions that result in a less invasive measurement procedure: in particular the absolute value of left ventricular volume can be substituted by the integral of the left ventricular outflow rate, and aortic pressure is used instead of left ventricular pressure since its value has to be known only around the end-systolic time.

The estimation problem concerning systemic vascular system parameters (a problem closely related to the one under consideration here), has been addressed, among many others in ( $\mathrm{Yu}$ et al., 1999; Yu et al., 1995), by using an extended Kalman filter. Most of these contributions consider single-beat estimation of maximum elastance.

In this paper a method is presented, for estimation of maximum ventricular elastance based on measurements extending over several beats. The approach is based on the use of a model of arterial load, connected to a time-varying elastance model of left ventricle. By means of such a dynamical model, properly extended to cover maximum elastance as a state variable, observability is studied, and a state observer is proposed, as an approach to estimation. This paper extended (Arabia et al., 2004), where a single beat algorithm is proposed, based on recursive least square, and (Borella et al., 2004), where a preliminary observability analysis has been carried out and results derived from an estimation algorithm based on Kalman filtering has been reported.

On the other side, the observability issue for hybrid and switched systems is a key one, together with the problem of observer design (see, among others, (Xie and Wang, 2004), (Ballucchi et al., 2003), (Bemporad et al., 2000) and the references therein).

The estimation make use either of ventricle volume and aortic pressure measurements, or of ventricle volume alone. Acquisition of these signals can be carried out in a wholly non invasive manner, e.g., via eco-graphic equipments as far as volume is concerned, and radial tonometry, together with a proper dynamic filter, as far as aortic pressure is concerned.

Simulation analysis carried out under a variety of conditions indicate good performance of the algorithm. 


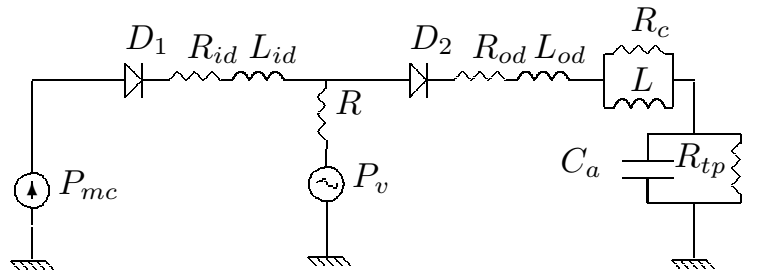

Fig. 1. Left heart model

\section{SYSTEM MODEL}

The left heart is modelled by an electrical-analog representation ( $\mathrm{LA} / \mathrm{LV} / \mathrm{AS}$ model), including the time-varying elastance model of the left ventricle by Suga and Sagawa (Suga et al., 1973; Suga and Sagawa, 1974), a simplified constant pressure model of the left atrium $\left(P_{a}\right)$, and the four parameters Burattini-Gnudi model of arterial load (Burattini and Gnudi, 1982) where $P_{l v}$ is the left ventricle pressure, $V_{l v}$ is the left ventricle volume, $R$ is the hydrodynamic resistance of the left ventricle, $Q_{e}$ is the ventricle input flow (see Fig.1).

The left ventricle pressure-volume (PV) relation is described by

$$
P_{l v}=E(t)\left(V_{l v}-V_{0}\right)+P_{0}
$$

where $P_{0}, V_{0}$ are the coordinates of a reference point and $\mathrm{E}(\mathrm{t})$ is the ventricular elastance, which begins to increase at the start of the cardiac cycle reaching a peak $\left(E_{\max }\right)$ at end-systole and decreases to a minimum value $\left(E_{\text {min }}\right)$ during diastole. The time-varying model used here is:

$$
E(t)=E_{\min }(1-h(t))+E_{\max } h(t)
$$

where $h(t)$ is a cosinusoidal standardized function:

$$
h(t)= \begin{cases}\frac{1}{2}\left[1-\cos \left(\frac{2 \pi t}{T_{i s o}}\right)\right] & 0<t \leq T_{\text {iso }} \\ 0 & T_{\text {iso }}<t<T_{c}\end{cases}
$$

with $T_{c}$ denoting the duration time of a cardiac cycle, and $T_{i s o}$ characterizing systolic duration.

Mitral and aortic valves are described by ideal diodes, in series with internal resistance $\left(R_{i d}\right.$ and $R_{o d}$, respectively) and internal inertance $\left(L_{i d}\right.$ and $L_{o d}$, respectively). Depending on the state of the two diodes, the systems is described by a different dynamic model, so that the overall systems turns out to be a switched system with four different discrete states, according to the following table 1 . Notice that the discrete state $\sigma=(1,1)$ does not usually occur, although could show up in some pathological conditions.

Simple application of the Kirkoff rules allow to derive the switched state space model of the system, described by:

$$
\dot{x}=f_{\sigma}(x)+g_{\sigma}(x) u(t),
$$

Table 1. Switched system discrete states

\begin{tabular}{cccl}
\hline$\sigma$ & Mitral valve & Aortic valve & Heart phase \\
\hline$\sigma=(0,0)$ & closed & closed & isovolumetric \\
$\sigma=(1,0)$ & open & closed & filling \\
$\sigma=(0,1)$ & closed & open & ejection \\
$\sigma=(1,1)$ & open & open & flow-through \\
& $x=\left(Q_{\text {ilv }}, V_{l v}, Q_{\text {olv }}, Q_{l}, P_{x}\right)$, & $u(t)=h(t) \quad(5)$
\end{tabular}

where the state vector $x$ comprises the mitral valve flow (ventricle input flow) $Q_{i l v}$, the ventricle volume $V_{l v}$, the aortic valve flow (ventricle output flow) $Q_{i l v}$, and the flow and pressure within the arterial load $Q_{l}$ and $P_{x}$. The input signal is the standardized function $h(t)$ given in $(3)$.

In the following, the ejection phase will play a special role, for the purpose of major interest $\mathrm{i}$ this paper, i.e., estimation of maximum elastance parameter $E_{\max }$. In this phase, the state space model is described by the following equation, corresponding to the electric analog depicted in figure 2:

$$
\begin{aligned}
\dot{x}_{1}= & 0 \\
\dot{x}_{2}= & -x_{3} \\
\dot{x}_{3}= & -\left(\frac{R_{o d}}{L_{o d}}+\frac{R_{G}}{L_{o d}}\right) x_{3}+E(t) \frac{x_{2}-V_{0}}{L_{o d}} \\
& +\frac{P_{o}}{L_{o d}}+\frac{R_{C}\left(x_{3}-x_{4}\right)+x_{5}}{L_{o d}} \\
\dot{x}_{4}= & \frac{R_{C}}{L}\left(x_{3}-x_{4}\right) \\
\dot{x}_{5}= & \frac{1}{C_{a}} x_{3}-\frac{1}{R_{t p} C_{a}} x_{5}
\end{aligned}
$$

Fig. 2. Ejection-phase left heart model

\section{OBSERVABILITY OF LA/LV/SA SYSTEM}

The parameter estimation approach proposed in this paper is based on the use of state observers for a properly extended system. The parameter of interest, namely maximum elastance $E_{\max }$, is assumed as an additional state variable. Then, observability of such an extended system clearly implies the possibility of identifying the parameter by means of an asymptotic observer.

The extended model is a switched system, and observability for this class of systems is subject of current interest from several researchers. In this 
paper, the basic idea is to verify observability of the sub-model corresponding to the ejection phase alone.

To this purpose, the electric analog of the cardiovascular model can be simplified as in Fig. 2 .

The observability analysis, carried out on the linear approximation, yield observability for output vector measurements comprising, among other, the following relevant ones:

$$
\begin{aligned}
& y_{1}=\left[P_{a o}, V_{l v}\right]^{T} \\
& y_{2}=\left[V_{l v}\right],
\end{aligned}
$$

where $P_{a o}$ denotes aortic pressure. Notice that such an analysis implies that maximum elastance can be estimated based solely on ventricle volume measurements.

On the contrary, a similar analysis shows that measurement of ventricle of aortic pressure alone does not yield observability.

The basic idea of the proposed asymptotic observer is to focus on ejection phase as for output injection, and to use the model corresponding to the other discrete states to propagate state estimate till the next ejection phase.

The structure of the observer is given, in compact form, as follows:

$$
\dot{\hat{x}}=f_{\sigma}(\hat{x})+g_{\sigma}(y) u(t)+L_{\sigma}(y-\hat{y}),
$$

where the input map $g_{\sigma}(y)$ turns out to depend solely on the ventricle volume, hence on output function, and $\hat{y}$ denotes output estimate based on current state estimate $\hat{x}$. As for output injection matrices $L_{\sigma}$, they are null in all the discrete state but the ejection one, corresponding to the discrete value $\sigma=(0,1)$. In this case, matrix $L_{(0,1)}$ is computed on the basis of the linear approximation of the corresponding dynamic model.

\section{SIMULATION EXPERIMENTS}

The proposed estimation method value has been evaluated with simulated data obtained through a Matlab model, based on cardiovascular parameter values available in literature.

The heart rate (HR) was set to 60 beats/min, with a sampling frequency $f_{s}=1 \mathrm{kHz}$.

The state and parameter estimator has been tested under both output function. For the sake of brevity, here only the case of ventricle volume measurement is reported. As the best of author knowledge, this is the first estimation algorithm not relying on pressure signals.

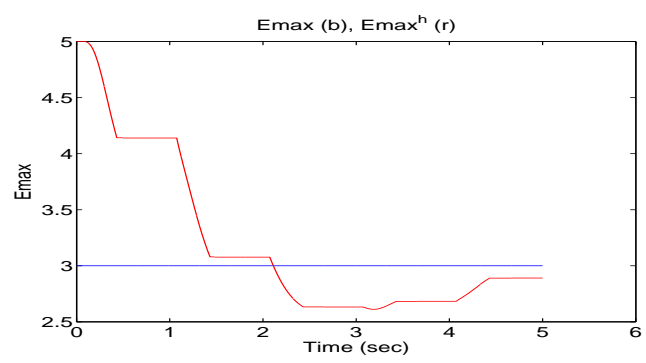

Fig. 3. Estimated maximum elastance (Pathological LV $E_{\max }=3$ )

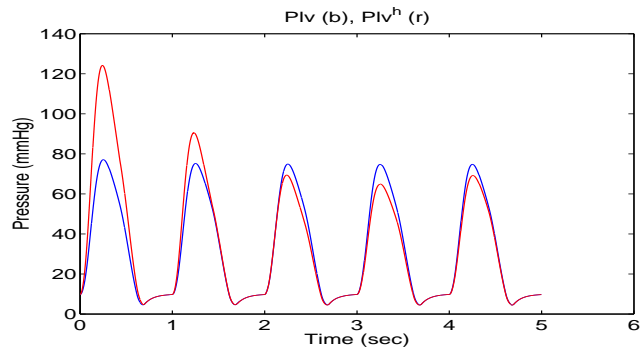

Fig. 4. Estimated ventricle pressure.

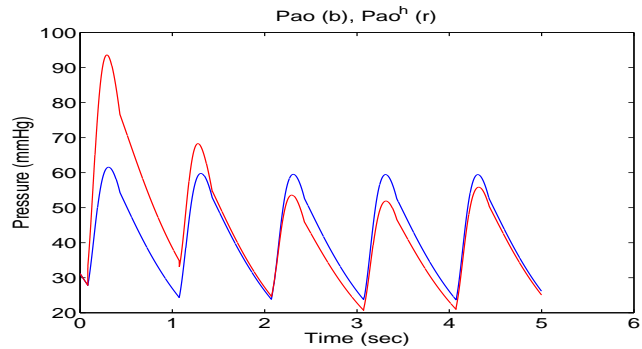

Fig. 5. Estimated aortic pressure.

Figures 3, 4 and 5 illustrate the behavior of the observer for a choice of injection matrix giving rise to a slow converging observer, which could be more robust to measurement noise. In particular, Fig. 3 illustrate convergence of $E_{\max }$ estimate, Fig. 4 convergence of ventricle pressure estimate and finally Fig. 5 illustrate aortic pressure estimate.

On the other side, figures 6,7 and 8 illustrate the behavior of the observer for a choice of injection matrix giving rise to a fast converging observer, which could be useful for short data sets. In particular, Fig. 6 illustrate convergence of $E_{\max }$ estimate, Fig. 7 convergence of ventricle pressure estimate and finally Fig. 8 illustrate aortic pressure estimate.

\section{CONCLUSIONS}

A method for estimating maximum ventricular elastance through an asymptotic observer for switched systems has been proposed. The algorithm is based on ventricular volume (and possibly aortic pressure) measurements, and is derived from an electrical-analog model of the left ventricle and systemic load. Based on such a model, 


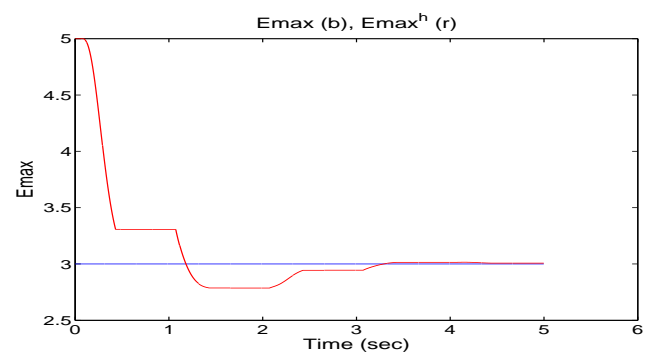

Fig. 6. Estimated maximum elastance (Pathological LV $E_{\max }=3$ )

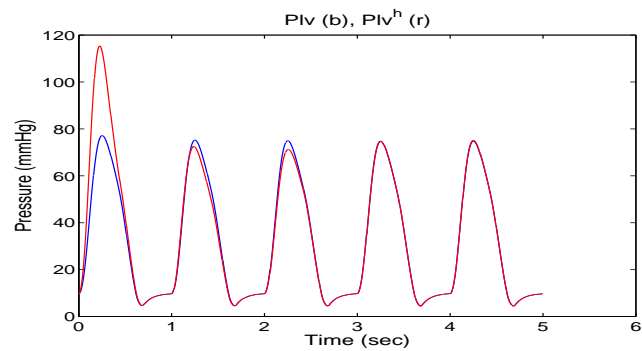

Fig. 7. Estimated ventricle pressure.

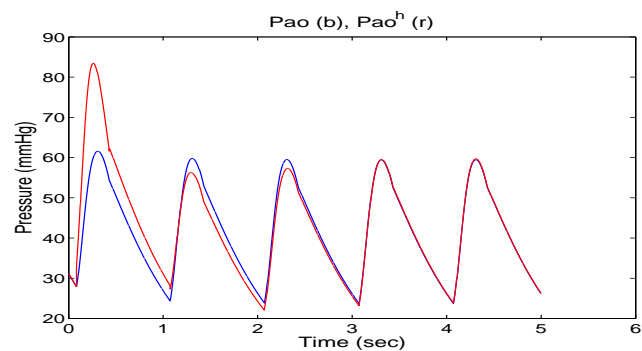

Fig. 8. Estimated aortic pressure.

restricted to the ejection phase, an observability study has been conducted.

Further issues to be addressed comprise estimation of systemic load parameters, and application to real data, as well as a more formal treatment of the problem of observer design for switched systems.

\section{REFERENCES}

Arabia, M., B. Borella, G. Danieli, E. Lucchi, S. Nicosia and P. Valigi (2004). The problem of estimating left ventricle maximum elastance. In: Proc. Conf. European Soc. Artificial Organs. Warsaw.

Arabia, Maurizio, Luca Boldrini and Paolo Valigi (2001). Afterload impedence feedback control of a failing left ventricle, assisted by pulsatile apical lvad. In: ESAO Int. Conf.. Gent, Belgium.

Ballucchi, A., L. Benvenuti, M. Di Benedetto and A.L. San Giovanni-Vincentelli (2003). Observability for hybrid systems. In: Proc. 42nd IEEE Int. Conference on Decision and Control. pp. 1159-1164.
Bemporad, A., G. Ferrari-Trecate and M. Morari (2000). Observability and controllability of piecewise affine and hybrid systems. IEEE Trans. Automatic Control 45(10), 1864-1876.

Boom, H.B.K. and H. Wijkstra (1989). Identification of left ventricular model parameters. In: Proc. 11th Annual Int. Conf. IEEE Engineering in Medicine and Biology Society, IEEE/EMBS. pp. 126-127.

Borella, Benedetta, Elena Lucchi, Salvatore Nicosia and Paolo Valigi (2004). A kalman filtering approach to estimation of maximum ventricle elastance. In: Proc. 26th Ann. Int. Conf. IEEE Engineering in Medicine and Biology Society, IEEE/EMBS. San Francisco.

Burattini, R. and G. Gnudi (1982). Computer identification of models for the arterial tree input impedance: Comparison between two new models and first experimental results. Med. Biol. Eng. Comput. 20, 134-144.

Guarini, M., J. Urzúa and A. Cipriano (1998). Estimation of cardiac function from computer analysis of the arterial pressure waveforms. IEEE Trans. Biomed. Eng. 45(12), 14201425 .

Kanai, H., S. Nakaya, H. Honda and Y. Koiwa (1999). Noninvasive estimation of left-ventricular end-diastole elasticity by analysing heart wall vibrations. Electronics Letters 35(10), 765-766.

Karamanoglu, M. and M.P. Feneley (1996). A non-invasive system for assessment of left ventricular systolic mechanical performance. In: Proc. 18th Annual Conf. IEEE Engineering in Medicine and Biology Society, IEEE/EMBS. Amsterdam. pp. 1324-1325.

Kim, K. M., D.D. Lee, J.K. Chung, M.C. Lee and Y.J. Kim (1998). Noninvasive measurement of left ventricular contractility using gated myocardial spect and arterial pressure tonometer. In: Proc. 20th Annu. Internat. Conf. IEEE Engineering in Medicine and Biology Society, IEEE/EMBS. Vol. 20. pp. 517519.

Nitta, S. and M. Yoshizawa (1995). A less invasive $E_{\max }$ estimation method for weaning from cardiac assistance. IEEE Trans. Biomed. Eng. 42(12), 1165-1173.

Ruggeri, A., A. Logiudice, W. Reiner and A. Erlicher (1997). A new approach for a minimally invasive measurement of cardiac contractility via ventricle elastance. In: Proc. of 19th Conf., IEEE Engineering in Medicine and Biology Society, IEEE/EMBS. Chicago. pp. 248-250.

Senzaki, H., C.H. Chen and D.A. Kass (1996). Single-beat estimation of end-systolic pressure- volume relation in humans. a new method with the potential for non invasive application. Circ. 94(10), 24972506 . 
Suga, H. and K. Sagawa (1974). Instantaneous pressure-volume relationships and their ratio in the excised, supported canine left ventricle. Circ. Res. 35, 117-126.

Suga, H., K. Sagawa and A.A. Shoukas (1973). Load independence of the instantaneous pressure-volume ratio of the canine left ventricle and effects of epinephrine and heart rate on the ratio. Circ. Res. 32, 314-322.

Xie, G. and L. Wang (2004). Necessary and sufficient condition for controllability and observability of switched impulsive control systems. IEEE Trans. Automatic Control 49(6), 960966.

Yoshizawa, M., A. Tanaka, K. Abe, H. Takeda, Y. Kakinuma, T. Yambe and S. Nitta (1998). Approach to single-beat estimation of $e_{\max }$ as an inverse problem. In: Proc.of 20th Conf., IEEE Engineering in Medicine and Biology Society, IEEE/EMBS. Vol. 20. pp. 379-382.

Yoshizawa, M., K. Abe, D. Sato, H. Takeda, T. Yambe and S. Nitta (1994). Less-invasive $e_{\max }$ estimation under cardiac assistance based on angular frequency method. In: Proc. 16th Ann. Int. Conf. IEEE Engineering in Medicine and Biology Society, IEEE/EMBS. Vol. 16. pp. 107-108.

Yoshizawa, M., S. Iemura, K. Abe, H. Takeda, Y. Kakinuma, H. Akiho, T. Yambe and S. Nitta (1996). Parameter optimization methodfor less-invasive and beat-by-beat estimation of $e_{\max }$. In: Proc.of 20th Conf., IEEE Engineering in Medicine and Biology Society, IEEE/EMBS. Amsterdam.

Yu, Y. C., J. R. Boston, M.A. Simaan and J.F. Antaki (1999). Sensitivity analysis of cardiovascular models for minimally invasive estimation of systemic vascular parameters. In: Proc. of American Control Conference. San Diego, CA. pp. 3392-3396.

Yu, Y.C., J.R. Boston, J. Antaki, M. Simaan and D.B. Breitenstein (1995). Parameter estimation in cardiovascular models. In: Proc. IEEE-EMBC Conf.. Montreal. pp. 14331434. 